\title{
Computer lessons for a social psychology research methods course
}

\author{
RUSSELL H. FAZIO and MARTIN H. BACKLER \\ Indiana University, Bloomington, Indiana 47405
}

\begin{abstract}
The development and use of three computer-based lessons for an undergraduate course on research methods in social psychology are described. One lesson is essentially a tutorial on main effects and interactions. Two additional lessons are simulations demonstrating the concepts of experimental power and survey sampling. A rationale and description of each lesson is provided.
\end{abstract}

The specific concerns that we hoped to address through the use of microcomputer-based lessons relate to an undergraduate course titled "Laboratory in Social Psychology." Essentially, the course introduces students to the wide variety of research methodologies (both laboratory and field) employed in the field of social psychology. The central long-term goal of the course is to acquaint the students with basic methodological principles so that students can evaluate whether an investigation is methodologically sound, interpret the data, and decide whether the conclusion should be accepted as stated.

Unfortunately, this goal is difficult to attain. Such concepts as power, interaction, internal validity, regression to the mean, and sampling are difficult for the beginning student to comprehend. Furthermore, methodology is not easily learned through lectures and the reading of text material. A solid grasp of the concepts is best attained by actual research experience. In this way, students can "learn by doing." Yet, time constraints typically preclude the use of such an approach, for even the simplest social psychological experiment requires weeks of data collection. Hence we have turned to microcomputer-based lessons as a means of exposing the students to, and involving them directly in, a variety of research methodologies, without expending a disproportionate amount of time on any single research project.

Three such lessons have been prepared. In each case, a specific methodological or data interpretation issue that the students frequently have difficulty understanding was identified. Furthermore, it was essential that the issue be one that was suited to computer application. In particular, the computer provided an advantage over handouts, in-class demonstrations, and the like, only if

This project was conceived while the first author was supported by a postdoctoral teaching fellowship from the Lilly Foundation. The project itself was supported by a grant from the EXXON Education Foundation. Requests for reprints can be addressed to Russell H. Fazio, Department of Psychology, Indiana University, Bloomington, Indiana 47405. M. H. Backler is with the Division of Development and Special Services. the issue was such that a lesson could benefit from the interactive capabilities of the computer.

The first lesson that we developed can be characterized as a tutorial on data interpretation. When examining data, students often find it difficult to discern whether two or more independent variables are exerting main and/or interactive effects upon the dependent variable. This lesson provides the students with practice at making such judgments. After discussing and illustrating factorial designs, main effects, and interactions, the lesson presents the student a series of data displays from two-factor designs. Either a table of means or a graph is displayed. The microcomputer then queries the student about the nature of the effects, delivers immediate feedback concerning the student's answers, and provides explanations regarding any errors the student had made. The frequent and immediate feedback provides the student with a clear indication of his/her level of understanding. After the series of two-factor designs, the student is exposed to a series of displays of three-factor designs. By the end of the lesson, the student has practiced judging whether the means from an experiment reveal main effects, two-way interactions, and/or threeway interactions. The value of this lesson was apparent from students' performance on exams. A class that predated the development of this lesson performed relatively poorly on an exam question concerned with main effects and interactions. The next year's class used the lesson, and all but 2 of the 18 students answered well enough to receive full credit on a similar question.

The other two lessons, power and sampling, make use of the computer to simulate research. Unlike the interactions lesson, which is largely tutorial in nature, these lessons allow the students to employ a "hypothesizeand-test" approach. Students are encouraged to run simulations based on their own choices and then immediately see the results. The computer takes over the mundane task of calculation and allows the lesson, and the student, to focus on the underlying concepts.

The first of these two simulations concerns the concept of power. In the course, we spend a considerable amount of time discussing how an experiment can be 
designed so as to enhance its power. Given the complexity of the concept and the myriad factors that affect power, students have difficulty comprehending power. The lesson allows students to run simulations of an experiment with or without various design improvements intended to minimize error variance and, hence, enhance power.

Stored in the computer is a data set generated to reflect the findings of an experiment on energy consumption conducted by Becker and Seligman (1978). The experiment examined the efficacy of a device that signaled homeowners that outside termperature had dropped sufficiently that they could cool the house effectively by turning off the air conditioner and opening the windows. Students can run simulations of an experiment comparing electrical consumption in homes in which the device was installed to that in homes in which it was not. The students are shown the mean electrical consumption in each condition, an F, and a $p$ value. They can compare the results of this basic experiment to experiments that incorporate various means of improving power. The students can increase $\mathrm{N}$, improve the reliability of the measures by lengthening the period of observation, increase the homogeneity of the sample by selecting only houses consisting of, for example, three-bedroom houses, include an important source of variation (e.g., two-, three-, or fourbedroom houses) as an additional factor in the design, create matched pairs of homes on the basis of insulation levels, and use repeated measures by also obtaining a pretreatment consumption level for each home. In this way, the students can see the benefits of increasing power that are achieved by a number of different steps that can be taken to minimize error variance.

The second simulation involves surveys and sampling. Stored in the computer are the data from a nationwide poll conducted by the Institute of Social Research (ISR) at the University of Michigan. The student imagines that he/she is a pollster who must sample the "population" residing within the computer. The student can select one of six questions concerned with such topics as nuclear power, school busing, and government spending to "ask" the sample. The student also specifies the .size of the sample and can employ either simple random sampling or stratified random sampling. Six demographic variables are available as possible stratifying variables. For each survey conducted, a sample is generated via the technique the student specified. The proportion of respondents who selected each response alternative is displayed, along with the confidence interval. The student can compare the precision of estimates across samples of varying size or across samples selected through simple random vs. stratified sampling. In this way, students can achieve a better understanding of how polls are conducted and of the determinants of estimate error rates.

Each of these lessons was developed with the goal that the computer be easy to use for a complete novice. A brief demonstration of the booting of the diskettes and of the use of the keyboard is all that is necessary for the students to approach the use of the Apple II without apparent apprehension.

We have consistently followed a policy of having the student complete an evaluation form after the use of any lesson. The form asks that students list any problems or ambiguities that arose as they worked the lesson and give their general reactions to the lesson. Responses to these questions provided helpful suggestions for subsequent revisions of the lessons. In addition to these openended questions, students respond to three 1-to 5-point scales on which they rated how valuable they found the lesson, how much they learned from the lesson, and how easy they found the computer to use. Of the 18 students who have used the lessons, $88.9 \%$ have checked scale points 4 or 5 with regard to the value of the lesson, indicating that they found it valuable. With regard to how much they learned from the lesson, $77.8 \%$ employed the upper two scale points. Finally, all 18 respondents circled the endpoint of the scale indicating the computer was very easy to use. Along with the examination results, these data suggest that the lessons are effective.

At present, the programs are available only in APPLESOFT BASIC using the DOS 3.3 operating system. The programs run on a 48-KB Apple II-Plus system. Only one disk drive is required. Anyone interested in obtaining a copy of any of the three lessons can send a blank disk (one disk per lesson) to the authors.

Since these lessons were developed in what may be an atypical manner, a brief comment about their development is warranted. A relationship that we suspect may be somewhat special and unique greatly contributed to the success of this project and may serve as a model for others interested in developing instructional software. The faculty member who wants to use specially designed instructional computer programs in a course faces a number of barriers. Unless he/she is a programmer and has a lot of free time, it is unlikely he will be able to carry out the project himself. An alternative is to find a source of funding and hire a programmer. However, the faculty member's lack of knowledge of what is possible and the programmer's lack of sophistication in instructional design may lead to disappointing results. Special authoring languages have been designed that are easy to learn. Unfortunately, these languages impose constraints on the lesson structure, making them inappropriate for many instructional applications.

At Indiana University, the Bloomington campus instructional consulting service, called the Division of Development and Special Projects (DDSP), provides a unique alternative for faculty with specific instructional needs. The mission of the division is to provide faculty with the expertise and resources necessary to achieve their instructional goals. In our case, a collaborative team of two people was created, a faculty member and an instructional consultant, each contributing special knowledge and skills crucial to the success of the project.

The faculty member provided the clear conception 
of the instructional need because of his previous experiences with the course and his knowledge of the students in the class. In addition, he contributed his subjectmatter expertise. The instructional consultant had skills in consulting and experience in instructional design. He was able to work together with the faculty member to define the need and design an appropriate solution. The consultant could make use of his knowledge of instructional options to suggest possible strategies to meet the instructional goals. In our case, the consultant was also the programmer, although this need not necessarily be the case. The programmer provided the skills necessary to turn the plan or script into a functioning computer program.

An important aspect of the relationship was its collaborative nature. The team members worked together through the course of the project, establishing a rapport that enhanced the chances of a successful outcome. An interactive relationship was established that accommodated the working styles of both members. Each person performed those tasks for which he had the expertise.

In the early stage of the project, the participants worked together to define the instructional needs and to lay out the strategic options for responding to them. In the next phase, scripts for the computer lessons were written, reviewed, and revised, taking into account sound instructional design principles and the capabilities of the computer. The revised script was then coded on the computer as a program. Once the lesson was running, it too was subjected to a cycle of reviews and revisions, using both students and the faculty member as evaluators. By working collaboratively, the team was able to generate effective computer lessons that were uniquely suited to the faculty member's needs.

\section{REFERENCE}

Becker, L. J., \& Seligman, C. Reducing air conditioning waste by signalling it is cool outside. Personality and Social Psychology Bulletin, 1978, 4, 412-415. 